\title{
Simulation of Monopole and Multipole Seismoelectric Logging
}

\author{
Zhiwen Cui, ${ }^{1}$ Jinxia Liu, ${ }^{1}$ Yujun Zhang, ${ }^{1}$ Kexie Wang, ${ }^{1}$ and Hengshan $\mathrm{Hu}^{2}$ \\ ${ }^{1}$ Department of Acoustics and Microwave Physics, College of Physics, Jilin University, Changchun 130012, China \\ ${ }^{2}$ Department of Astronautics and Mechanics, Harbin Institute of Technology, Harbin 150001, China
}

Correspondence should be addressed to Zhiwen Cui, cuizw@jlu.edu.cn

Received 14 September 2010; Accepted 13 January 2011

Academic Editor: Fidel E. Hernandez Montero

Copyright () 2011 Zhiwen Cui et al. This is an open access article distributed under the Creative Commons Attribution License, which permits unrestricted use, distribution, and reproduction in any medium, provided the original work is properly cited.

\begin{abstract}
In a fluid-saturated porous formation, acoustics and electromagnetic waves are coupled based on Pride seismoelectric theory. An exact treatment of the nonaxisymmetric seismoelectric field excited by acoustic multipole sources is presented. The frequency wavenumber domain representations of the acoustic field and associated seismoelectric field due to acoustic multipole sources are formulated. The full waveforms of acoustic waves and electric and magnetic fields in the time domain propagation in borehole are simulated by using discrete wave number integration, and frequency versus axial-wave number responses are presented and analyzed.
\end{abstract}

\section{Introduction}

The study of wave propagation in a fluid-saturated porous medium is of considerable interest in acoustics and geophysics due to its important applications in various technical and engineering processes. The investigation of wave propagation in fluid-saturated porous media was early developed by Biot $[1,2]$. One of the major findings of Biot's theory was that there is a compressional slow wave in a fluidsaturated porous medium. The first clear experimental observation of this slow wave was reported by Plona [3]. Biot predicted the slow waves should have an important bearing on electrokinetic effect [4]. This predication has been quantitatively confirmed by Pride [5] and $\mathrm{Hu}$ [6]. Elastic waves propagating in fluid-saturated porous media generate a movement of the ions in the pore fluid. Such movement induces an electromagnetic (EM) field. Thompson and Gist [7] have made field measurement clearly demonstrating that seismic waves can induce electromagnetic disturbances in saturated sediments. Pride [8] derived the governing equations for the coupled acoustics and electromagnetic waves in fluid-saturated porous media. Pride and Haartsen [9] analyzed the basic properties of seismoelectric waves.

The electric field induced by elastic waves is weak and attenuates in propagation. In order to detect the seismoelectric signal effectively, Haartsen and Pride [10] suggested measuring vertical electroseismic profile. Mikhailov et al. [11] measured the electric field converted from low frequency Stoneley waves in a borehole and made theoretical analysis. Seismoelectric logging method has been proposed to detect deep target formation. The advantage of seismoelectric logging is the distance both from transmitter to target formation and from the transmitter to the receiver is small, and signals can be received with relative high amplitude. Zhu et al. [12-14] made laboratory experiments and observed the seismoelectric conversion in model wells, and their experimental results confirm that seismoelectric logging could be a new borehole logging technique. Seismoelectric logging methods based on the excitation and reception of axisymmetric wave phenomena in a fluid-filled borehole embedded in permeable formations have been investigated $[6,15]$. In the present paper, we consider the nonaxisymmetric seismoelectric field excited by acoustic multipole sources based on the entire Pride seismoelectric theory [9]. We theoretically formulate and numerically simulate both axisymmetric and nonaxially symmetric waves in a fluid-filled borehole surrounded by a homogeneous fluidsaturated porous formation. The frequency versus axial-wave number responses are presented, and time-domain transient waveforms of acoustic waves and electromagnetic waves propagation in borehole are simulated and compared. The current model may be an important tool in understanding and predicting borehole seismoelectric phenomena. This paper may be of interest for researchers in the field referred 


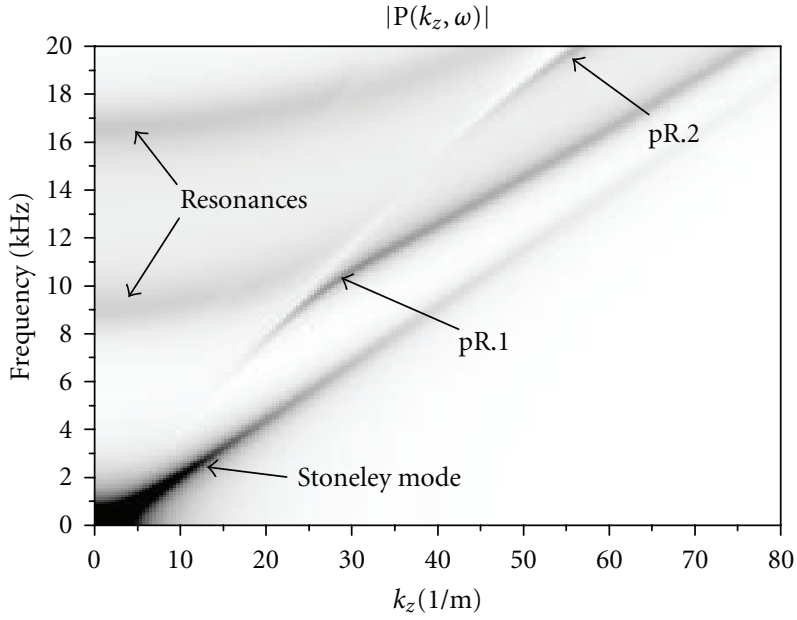

(a)

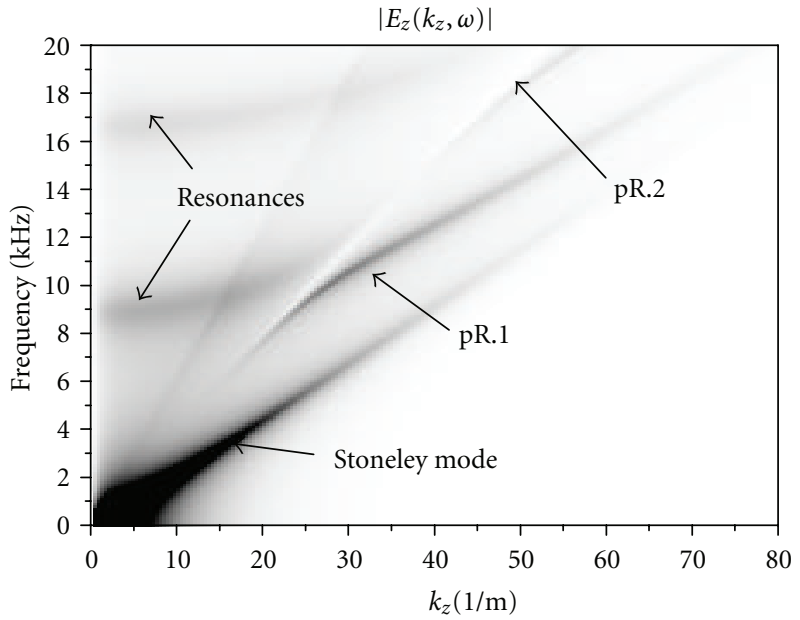

(c)

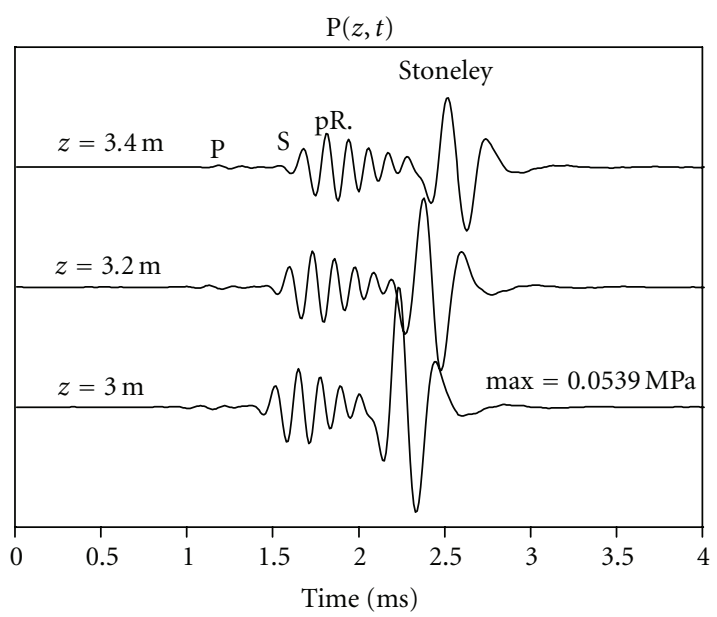

(b)

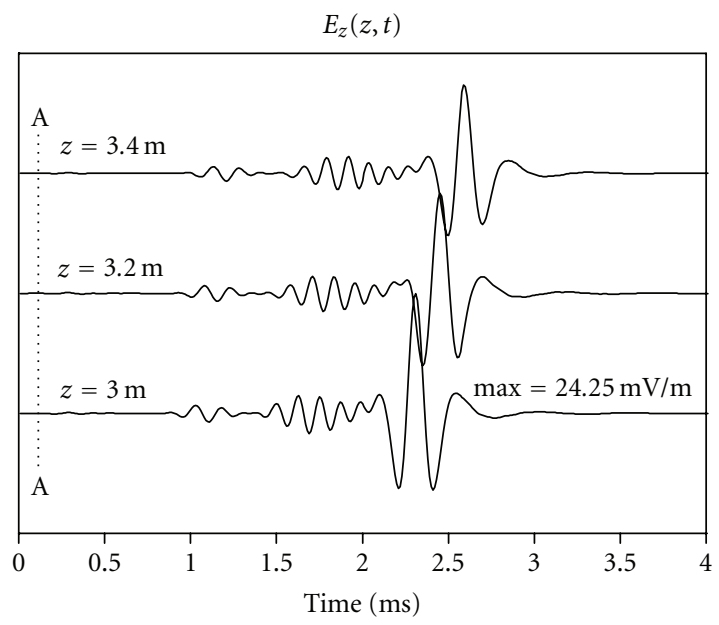

(d)

FIGURE 1: Spectral representation and time response of borehole pressure and electric fields generated by monopole source. (a) and (c) are spectral of pressure and electric fields, respectively. (b) and (d) are time responses of pressure and electric fields, respectively.

to above as well as experimental wave propagation in fluidsaturated porous media.

\section{Theoretical Formulations}

In this section we formulate a frequency wavenumber integral representation for the acoustic, electric, and magnetic fields excited by a multipole acoustic source in the borehole fluid. Consider a fluid-filled borehole of radius $a$ embedded in linear isotropic fluid-saturated porous medium and acoustic multipole sources in the borehole. In the borehole, the acoustic and electromagnetic fields are not coupled, but when the acoustic source in the borehole is excited, according to Pride's theory $[8,9]$, the acoustic waves in porous formation will generate accompanying electric and magnetic fields, and acoustic wave induces radiating EM wave at borehole wall (discontinuous interface). We can receive electric or magnetic fields signal inside the borehole due to the boundary condition at the borehole wall.
2.1. The Acoustic Field in the Borehole Fluid. We consider acoustic multipole sources inside the borehole. The Dipole source is constructed from two point sources of opposite sign placed close together in the same horizontal plane. Equivalently, the dipole can be viewed as a point force oriented horizontally and pointed at an azimuthal angle of $\theta_{0}$. Similarly, a quadrupole source can be viewed as two closely spaced dipole sources pointing in opposite directions. Higher-order multipoles have similar interpretations. Following Kurkjian and Chang [16], with emphasizing the radius of a multipole source being very small and $\left|\eta_{f} r_{0}\right| \ll$ 1 , the representation in the frequency-wavenumber domain of the displacement potential associated with $n$ acoustic multipole sources $\left(r>r_{0}\right)$ is given by

$$
\Phi_{0}\left(r, \theta, k_{z}, \omega\right)=\gamma \varepsilon_{n} K_{n}\left(\eta_{f} r\right) \cos n\left(\theta-\theta_{0}\right),
$$

where $\gamma=V_{0}(\omega)\left(\eta_{f} r_{0} / 2\right)^{n} / n !, \varepsilon_{n}$ is Neumann's factor $\left(\varepsilon_{n}=1\right.$ for $n=0 ; \varepsilon_{n}=2$ for $n=$ otherwise), $V_{0}(\omega)$ is the source 


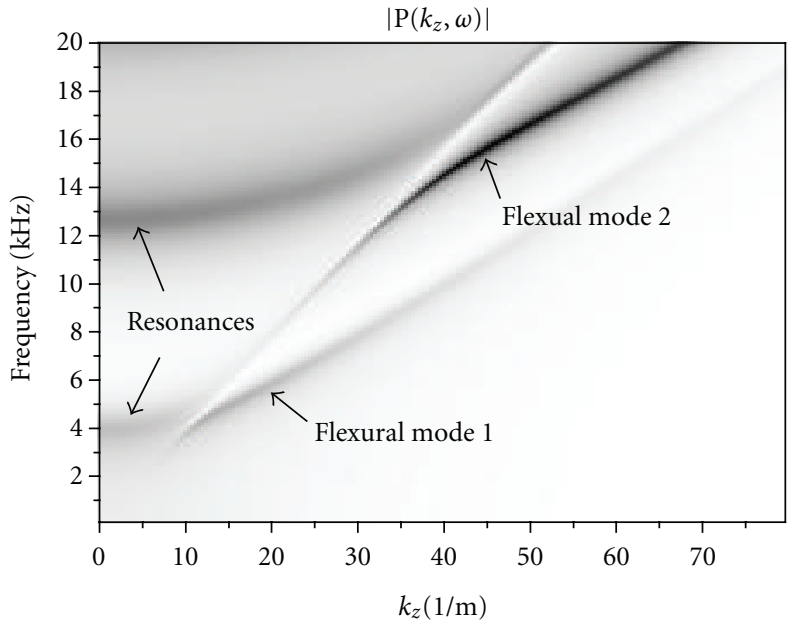

(a)

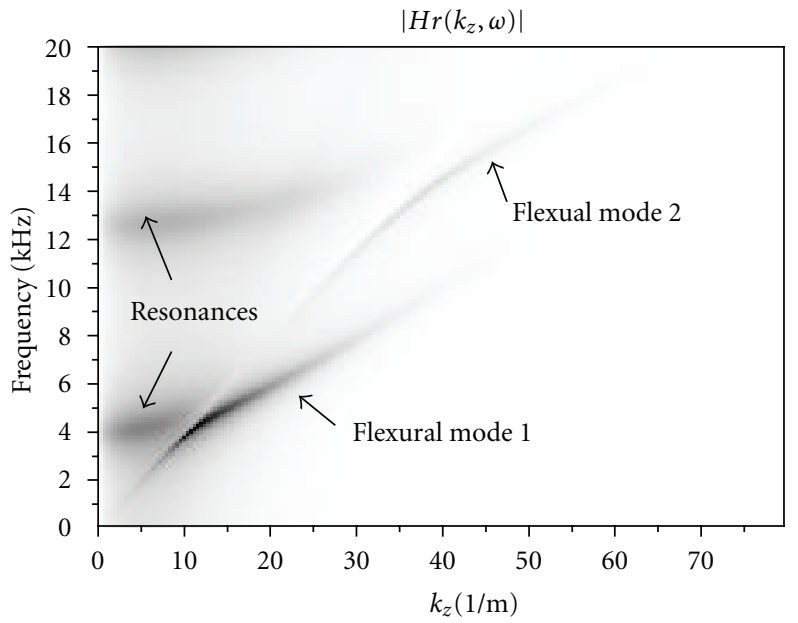

(c)

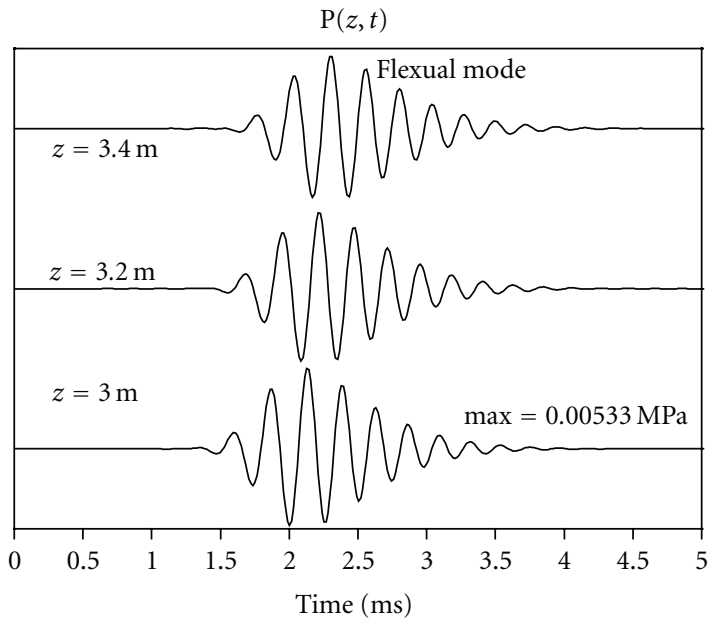

(b)

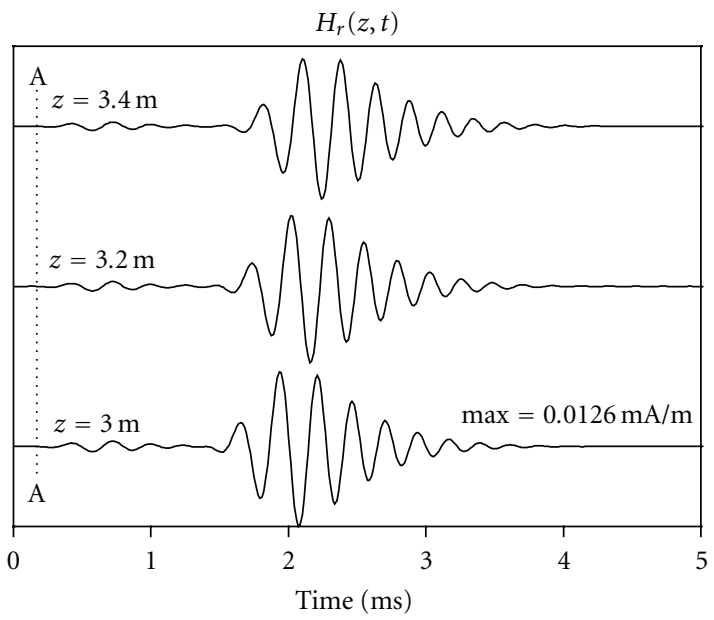

(d)

FIGURE 2: Spectral representation and time response of borehole pressure and magnetic fields for fast formation generated by dipole source. (a) and (c) are spectral of pressure and magnetic field respectively. (b) and (d) are time responses of pressure and magnetic field respectively.

spectrum, and $\eta_{f}^{2}=k_{z}^{2}-\omega^{2} / c_{f}^{2}$ is the radial wavenumber with respect to the acoustic velocity of bore fluid $c_{f} . K_{n}(x)$ is the $n$th order modified Bessel function of the second kind.

The total acoustic wave field in the borehole is the sum of the source contribution and the reflected acoustic field. The radiation conditions prescribe that the reflected field should be finite at the axis of the borehole, thus the reflected waves displacement potential associated with $n$ acoustic multipole sources is

$$
\Phi_{1}\left(r, \theta, k_{z}, \omega\right)=\gamma A_{n} I_{n}\left(\eta_{f} r\right) \cos n\left(\theta-\theta_{0}\right)
$$

where $I_{n}(x)$ is the $n$ th-order modified Bessel function of the second kind. Thus, the total field inside the borehole in the frequnecy-wavenumber domain is given by

$$
\Phi\left(r, \theta, k_{z}, \omega\right)=\gamma\left[A_{n} I_{n}\left(\eta_{f} r\right)+\varepsilon_{n} K_{n}\left(\eta_{f} r\right)\right] \cos n\left(\theta-\theta_{0}\right),
$$

the reflection coefficients $A_{n}$ can be derived by the boundary conditions at the borehole wall. The radial component of the displacement field in the borehole fluid is given by $u_{r}=$ $\partial \Phi / \partial r$ and the radial component of normal stress in the borehole fluid is equal to the negative of borehole fluid pressure, that is,

$$
\tau_{r r}=-P\left(r, \theta, k_{z}, \omega\right)=-\rho_{f} \omega^{2} \Phi .
$$

2.2. The Electromagnetic Field in the Borehole Fluid. In cylindrical coordinate system $(r, \theta, z)$, it is convenient for us to take the $\mathbf{z}$-component of vector $\mathbf{E}$ and $\mathbf{H}$, and they satisfy the Homheltz-type wave equations without electric current sources in the borehole,

$$
\begin{aligned}
\nabla^{2} E_{z}+k_{e}^{2} E_{z} & =0, \\
\nabla^{2} H_{z}+k_{e}^{2} H_{z} & =0,
\end{aligned}
$$

where $k_{e}^{2}=\omega^{2} \mu \varepsilon_{b}, \varepsilon_{b}=\varepsilon_{f}\left(1+i \sigma_{f} / \omega \varepsilon_{f}\right), \varepsilon_{f}$ is the borehole fluid's electrical permittivity, $\sigma_{f}$ is the borehole fluid's 


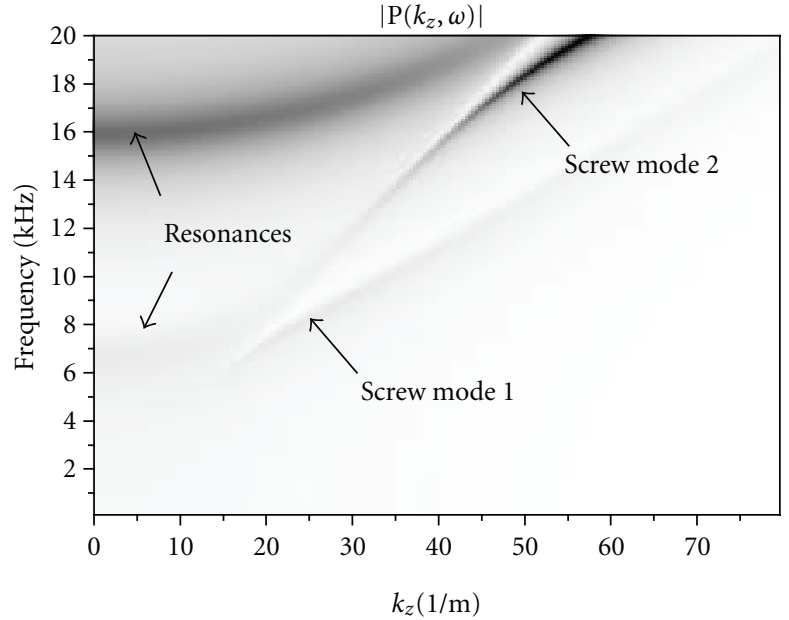

(a)

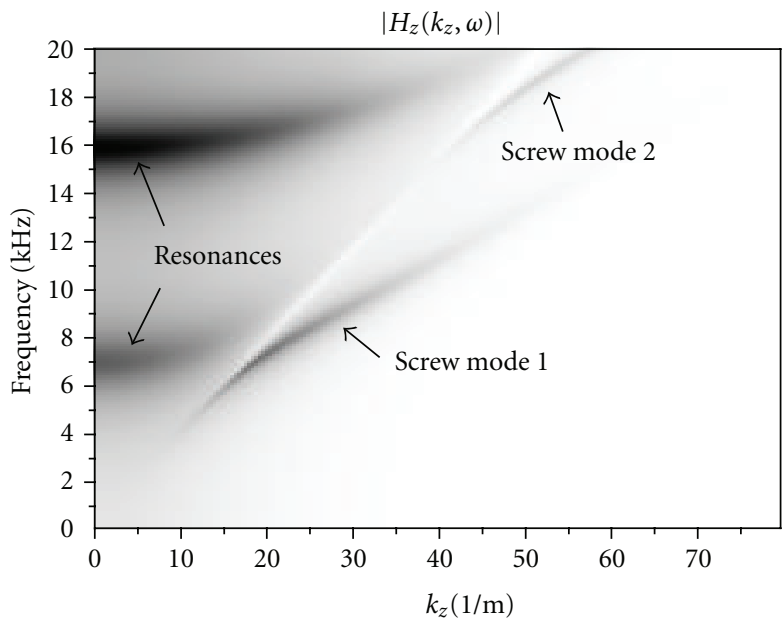

(c)

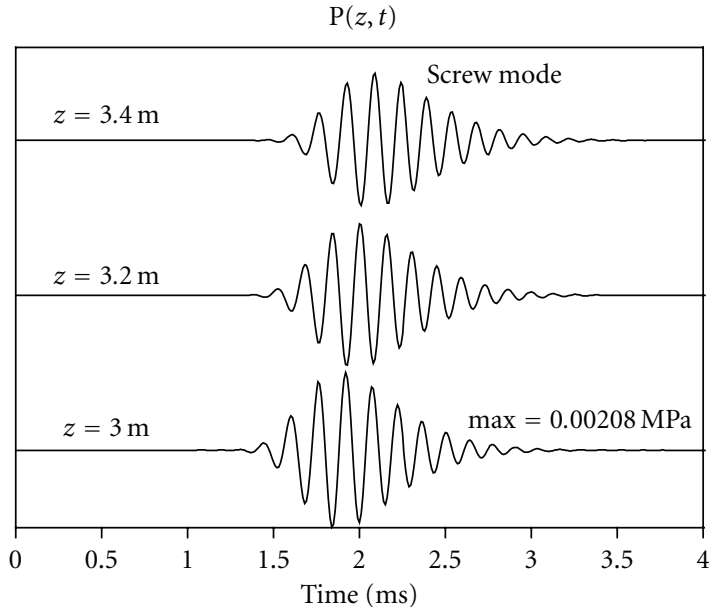

(b)

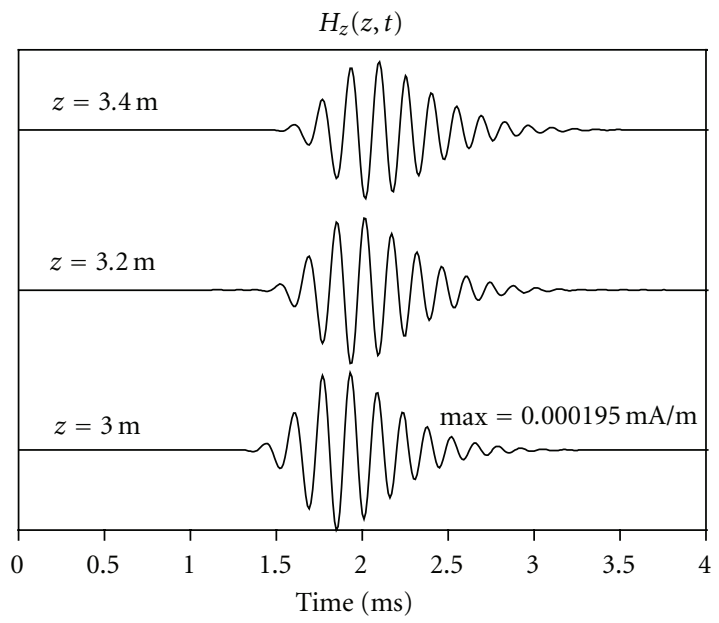

(d)

FIgURE 3: Spectral representation and time response of borehole pressure and magnetic fields for fast formation generated by quadrupole source. (a) and (c) are spectral of pressure and magnetic fields respectively. (b) and (d) are time responses of pressure and magnetic fields respectively.

electrical conductivity, and $\mu$ is the magnetic permeability. For really fluid and all porous media of interest it can be assumed that $\mu=\mu_{0}$. In borehole fluid and fluid-saturated porous media we take $\mu=\mu_{0}$ throughout the paper.

Considering the radiation conditions, that prescribe the reflected electromagnetic field should be finite at the axis of the borehole, we can take the solutions for $E_{z}$ and $H_{z}$ as

$$
\begin{aligned}
E_{z}\left(r, \theta, k_{z}, \omega\right) & =\gamma A_{n}^{E} I_{n}\left(\eta_{f} r\right) \cos n\left(\theta-\theta_{0}\right), \\
H_{z}\left(r, \theta, k_{z}, \omega\right) & =\gamma A_{n}^{H} I_{n}\left(\eta_{f} r\right) \sin n\left(\theta-\theta_{0}\right),
\end{aligned}
$$

where $\eta_{e}^{2}=k_{z}^{2}-k_{e}^{2}$. As we will see, in order to satisfy the boundary conditions of continuity of tangential electromagnetic fields at borehole wall $r=a$, the solutions in general require a combination of both TE and TM fields. The modes with $E_{z}$ and $H_{z}$ components present are hybrid modes.
Transverse field components are obtained from Maxwell's equations,

$$
\begin{aligned}
E_{\theta} & =\frac{i}{\eta_{e}^{2}}\left(\omega \mu \frac{\partial H_{z}}{\partial r}-\frac{k_{z}}{r} \frac{E_{z}}{\partial \theta}\right), \\
H_{\theta} & =\frac{-i}{\eta_{e}^{2}}\left(\omega \varepsilon_{b} \frac{\partial E_{z}}{\partial r}+\frac{k_{z}}{r} \frac{\partial H_{z}}{\partial \theta}\right), \\
E_{r} & =\frac{-i}{\eta_{e}^{2}}\left(\frac{\omega \mu}{r} \frac{\partial H_{z}}{\partial \theta}+k_{z} \frac{\partial E_{z}}{\partial r}\right), \\
H_{r} & =\frac{i}{\eta_{e}^{2}}\left(\frac{\omega \varepsilon_{b}}{r} \frac{\partial E_{z}}{\partial \theta}-k_{z} \frac{\partial H_{z}}{\partial r}\right),
\end{aligned}
$$

Reflected electromagnetic field inside the borehole in the time-space domain is given by

$$
\begin{aligned}
& \Pi(r, \theta, z, t) \\
& \quad=\frac{1}{2 \pi} \iint_{\infty}^{\infty} \Pi\left(r, \theta, k_{z}, \omega\right) \exp \left[i\left(k_{z} z-\omega t\right)\right] d k_{z} d \omega ;
\end{aligned}
$$


here we use $\Pi$ to denote any component of electric or magnetic field given by (7)-(12). The reflection coefficients $A_{n}^{E}, A_{n}^{H}$ can be derived by the boundary conditions at the borehole wall.

2.3. Seismoelectric Waves in the Porous Formation. For the problem of modeling the propagation of coupled electromagnetic and mechanical disturbances in an isotropicporous material, Pride [8] has derived equations that control such "seismoelectric" phenomena. According to Pride's theory, without the applied force and electric current sources existing, and assuming an time dependence $\exp (-i w t)$, we may write the equations for the coupled electromagnetic and acoustics in macroscopically homogenous, isotropic, fluid saturated porous media as follows

$$
\begin{gathered}
\nabla \cdot \boldsymbol{\tau}=-\omega^{2}\left(\rho \mathbf{u}+\rho_{f} \mathbf{w}\right), \\
\boldsymbol{\tau}=\left(H-2 G_{b}\right)(\nabla \cdot \mathbf{u}) \mathbf{I}+C(\nabla \cdot \mathbf{w}) \mathbf{I} \\
+G_{b}\left(\nabla \mathbf{u}+\nabla \mathbf{u}^{T}\right), \\
-p_{f}=C(\nabla \cdot \mathbf{u})+M(\nabla \cdot \mathbf{w}), \\
-i \omega \mathbf{w}=\frac{\left(-\nabla p_{f}+\rho_{f} \omega^{2} \mathbf{u}\right) k(\omega)}{\eta}+L(\omega) \mathbf{E}, \\
\mathbf{J}=\left(-\nabla p+p_{f} \omega^{2} \mathbf{u}\right) L(\omega)+\sigma(\omega) \mathbf{E}, \\
\nabla \times \mathbf{E}=i \omega \mu \mathbf{H}, \\
\nabla \times \mathbf{H}=-i \omega \varepsilon \mathbf{E}+\mathbf{J},
\end{gathered}
$$

Those governing equations are the Biot equations for porous media acoustic along with the Maxwell equations for the electric and magnetic fields $\mathbf{E}$ and $\mathbf{H}$. Here $\boldsymbol{\tau}$ is the bulk stress in the porous medium, $p_{f}$ is the pressure in the pore fluid, $\mathbf{u}$ is the displacement in the solid, and $\mathbf{w}$ is the relative fliud-solid motion. The symbol $\rho$ denotes the bulk density of the porous medium, $\rho=(1-\phi) \rho_{s}+\phi \rho_{f}, \phi$ is the porosity of the medium, $\rho_{s}$ is the solid density, and $\rho_{f}$ denotes the fliud density. Equations (17) and (18) are in the form of Darcy law and Ohm law, through which acoustic and electromagnetic fields are coupled, where $\mathbf{J}$ is the electriccurrent density and $-i \omega \mathbf{w}$ is the Darcy filtration velocity. Where $H, C, M$, and $G_{b}$ are four moduli of isotropic porous media. As the most important coefficient is set to zero, Pride's equations will be separated into Biot's equations for elastic field and Maxwell equations for electromagnetic field. Here $\sigma(\omega)$ is the frequency-dependent electrical conductivity of the medium, $k(\omega)$ is the dynamic permeability, $\eta$ is the fluid dynamic viscosity, and $L(\omega)$ is the frequency-dependent electrokinetic coupling coefficient.The expressions for $\sigma(\omega)$, $k(\omega)$, and $L(\omega)$ are given in Pride [8].

The solution to the coupled equations of seismoelectric waves motions can be obtained by separating the basic field $\mathbf{u}, \mathbf{w}, \mathbf{E}$ into its compressional, and vertically and horizontally polarized shear components, and written in terms of scalar potential functions as

$$
\begin{aligned}
\mathbf{u}= & \nabla\left(\Phi_{p f}+\Phi_{p s}\right)+\nabla \times\left(\Psi_{s h}+\Psi_{e m}\right) \mathbf{e}_{z} \\
& +\nabla \times \nabla \times\left(\Gamma_{s h}+\Gamma_{e m}\right) \mathbf{e}_{z} \\
\mathbf{w}= & \nabla\left(\alpha_{p f} \Phi_{p f}+\alpha_{p s} \Phi_{p s}\right)+\nabla \times\left(\alpha_{s h} \Psi_{s h}+\alpha_{e m} \Psi_{e m}\right) \mathbf{e}_{z} \\
& +\nabla \times \nabla \times\left(\alpha_{s h} \Gamma_{s h}+\alpha_{e m} \Gamma_{e m}\right) \mathbf{e}_{z} \\
\mathbf{E}= & \nabla\left(\beta_{p f} \Phi_{p f}+\beta_{p s} \Phi_{p s}\right)+\nabla \times\left(\beta_{s h} \Psi_{s h}+\beta_{e m} \Psi_{e m}\right) \mathbf{e}_{z} \\
& +\nabla \times \nabla \times\left(\beta_{s h} \Gamma_{s h}+\beta_{e m} \Gamma_{e m}\right) \mathbf{e}_{z},
\end{aligned}
$$

where $\Phi_{j}$ is the compressional wave potential, $\mathbf{e}_{z}$ is the unit vector in the $z$ direction, $\Psi_{j}$ is the horizontal polarized shear potential, and $\Gamma_{j}$ is vertically polarized shear wave potential. Each of these potentials satisfy Helmholtz-type wave equation. The factors $\alpha_{p f}, \alpha_{p s}, \alpha_{s h}, \alpha_{e m}, \beta_{p f}, \beta_{p s}, \beta_{s h}$, and $\beta_{\text {em }}$ as well as the formulas of the body wave velocities can be found in $[9,15,17]$.

Outside the borehole, because the radiation conditions prescribe no incoming wave existing at the position where $r$ tends to infinity, the solutions in the frequency-axial wavenumber with respect to an $n$ th-order multipole source are, similar to only acoustic fields case $[18,19]$,

$$
\begin{aligned}
& \Phi_{j}\left(r, \theta, k_{z}, \omega\right)=\gamma B_{n}^{j} K_{n}\left(\eta_{j} r\right) \cos n\left(\theta-\theta_{0}\right), \\
& \Psi_{j}\left(r, \theta, k_{z}, \omega\right)=\gamma C_{n}^{j} K_{n}\left(\eta_{j} r\right) \cos n\left(\theta-\theta_{0}\right), \\
& \Gamma_{j}\left(r, \theta, k_{z}, \omega\right)=\gamma D_{n}^{j} K_{n}\left(\eta_{j} r\right) \cos n\left(\theta-\theta_{0}\right),
\end{aligned}
$$

where $\eta_{j}^{2}=k_{z}^{2}-k_{j}^{2}\left(k_{j}^{2}=\omega^{2} s_{j}^{2}\right)$ are the radical wavenumbers of the seismoelectric waves whose complex slowness $s_{j}(j=p f, p s, s h, e m)$ expressions can be found in $[9,15,17]$. Here, $B_{n}^{j}, C_{n}^{j}$, and $D_{n}^{j}$ which characterize the outgoing seismoelectric waves, are functions of the axial wavemumber $k_{z}$ and the angular frequency $\omega$. Once $\mathbf{u}, \mathbf{w}$, $\mathbf{E}$ are known, all other quantities such as stress tensor, pore fluid pressure, and magnetic vector can be deduced by (15)(16) and (19). The components of seisoelectric filed in the formation can be written in terms of the potentials using (23) and (26). The detailed expressions of those formulations can be found in [17].

2.4. Boundary Conditions and Resolution. The unknown coefficients $A_{n}, A_{n}^{E}, A_{n}^{H}, B_{n}^{p f}, B_{n}^{p s}, C_{n}^{s h}, C_{n}^{e m}, D_{n}^{s h}$, and $D_{n}^{e m}$ which appear in the expressions for the field potentials are determined by the boundary conditions. The boundary conditions of seismoelectric waves for a cylindrical interface (at $r=a$ ) between a fluid and a fluid-saturated porous media 
for the case of permeable interface are

$$
\begin{gathered}
u_{r}^{I}=u_{r}^{I I}+w_{r}^{I I}, \quad-P^{I}=\tau_{r r}^{I I}, \quad 0=\tau_{r z}^{I I} ; \\
0=\tau_{r \theta}^{I I}, \quad P^{I}=p_{f}^{I I} ; \\
E_{\theta}^{I}=E_{\theta}^{I I}, \quad E_{z}^{I}=E_{z}^{I I}, \quad H_{\theta}^{I}=H_{\theta}^{I I}, \quad H_{z}^{I}=H_{z}^{I I},
\end{gathered}
$$

where the superscript $I$ and $I I$ represent the media inside and outside the borehole, respectively. In the present paper, we do not consider the possibility of a sealed or partially sealed boundary condition. With the expressions of $w_{r}, u_{r}, \tau_{r r}, \tau_{r z}$, $\tau_{r \theta}, E_{\theta}, E_{z}, H_{\theta}, H_{z}$ deduced from (14)-(26), (7) -(10), and $u_{r}^{I}=\partial\left(\Phi_{0}+\Phi_{1}\right) / \partial r, P^{I}=\rho_{f} \omega^{2}\left(\Phi_{0}+\Phi_{1}\right)$ boundaries $(27)$ may be written in a matrix form as

$$
\bar{M} A=B
$$

where $\bar{M}$ are $9 \times 9$ complex matrices whose elements are given in the [17] and

$$
\begin{gathered}
A=\left\{A_{n}, B_{n}^{p f}, B_{n}^{p s}, C_{n}^{s h}, D_{n}^{s h}, C_{n}^{e m}, D_{n}^{e m}, A_{n}^{E}, A_{n}^{H}\right\}^{T} \\
B=\left\{b_{1}, b_{2}, 0,0, b_{5}, 0,0,0,0\right\}^{T}
\end{gathered}
$$

where $b_{1}=\varepsilon_{n}\left[\eta_{f} K_{n+1}\left(\eta_{f} a\right)-(n / a) K_{n}\left(\eta_{f} a\right)\right], b_{2}=b_{5}=$ $-\rho_{f} \omega^{2} \varepsilon_{n} K_{n}\left(\eta_{f} a\right)$.

\section{Simulations}

In this section, we present synthetic acoustic waves and electromagnetic field in the borehole excited by acoustic monopole, dipole, and quadrupole sources. The formation in our numerical example is permeable fluid saturated porous media and the medium input parameters describing the porous formation are given in Table 1. The borehole radius $a$ is $0.1 \mathrm{~m}$. The same medium parameters and source function as in [20]. The source is a cosine pulse [19]. If set acoustic transducer, electrode, and magnetometer in the borehole fluid, one could measure acoustic, electric and magnetic fields, respectively. For acoustic logging, only the axisymmetric modes contribute to the pressure on the axis [21]. However, for electroacoustic logging, beside axisymmetric modes, dipole modes of nonaxisymmetric modes also contribute to the electric and magnetic field on the axis $[20,22]$. For example, we only select some position $(0.05,0, z)$ off the axis to record the full waveforms. The spectral response is calculated similar to [23].

3.1. Fast Formation. Figure 1 shows spectral response and synthetic waveforms of acoustic waves and electric field for fast formation generated by an acoustic monopole source. In our calculation, the source is set to be pressure source, and the center frequency is $6 \mathrm{kHz}$. The pressure amplitude is $10 \mathrm{MPa}$ measured at $1 \mathrm{~cm}$ away from the source center. The numerical result of borehole pressure waveforms is on the order of those by Kurkjian [16]. From Figures 1(b) and 1(d), we can find there are four wave groups in electric field $E_{z}$ that is different from three in acoustic waveforms. A clear
TABLE 1: Medium properties used at input for numerical calculations.

\begin{tabular}{lccc}
\hline Property & $\begin{array}{c}\text { Fast } \\
\text { formation }\end{array}$ & $\begin{array}{c}\text { Slow } \\
\text { formation }\end{array}$ & $\begin{array}{c}\text { Borehole } \\
\text { fluid }\end{array}$ \\
\hline Porosity $\phi$ & 0.2 & 0.2 & - \\
Permeability $\kappa_{0}, \mu \mathrm{m}^{2}$ & 1.0 & 1.0 & - \\
Tortuosity $\alpha_{\infty}$ & 3.0 & 3.0 & - \\
Bulk modulus solid & $35.70 \times 10^{9}$ & $33.40 \times 10^{9}$ & - \\
$K_{s}, P a$ & & & \\
Bulk modulus fluid & $2.25 \times 10^{9}$ & $2.25 \times 10^{9}$ & $2.25 \times 10^{9}$ \\
$K_{f}, P a$ & & & \\
Frame bulk modulus & $14.39 \times 10^{9}$ & $2.61 \times 10^{9}$ & - \\
$K_{b}, P a$ & & & \\
Frame shear modulus & $13.99 \times 10^{9}$ & $2.83 \times 10^{9}$ & - \\
$G_{b}, P a$ & & & \\
Density solid $\rho_{s}$, & 2650 & 2650 & - \\
Kg/m ${ }^{3}$ & & & \\
Density fluid $\rho_{f}$, & 1000 & 1000 & 1000 \\
Kg/m ${ }^{3}$ & & 0.001 & 0.001 \\
Fluid viscosity $\eta$, Pa.s & 0.001 & 0.01 & 0.01 \\
Salinity $C_{0}$, mol/L & 0.01 & $80 \varepsilon_{0}$ & $80 \varepsilon_{0}$ \\
Permittivity of fluid & $80 \varepsilon_{0}$ & $4 \varepsilon_{0}$ & - \\
$\varepsilon_{f}$ & & & \\
Permittivity of solid & $4 \varepsilon_{0}$ & & \\
$\boldsymbol{\varepsilon}_{s}$ & & & \\
\hline
\end{tabular}

seismoelectric response A-A group for the electric field is relatively weak but visible in full waveforms, which were recorded at all receivers simultaneously. And the following groups for the electric field having apparent velocities are comparable to those of borehole acoustic waves. Because of the nonequilibrium of free charges in the pore fluid at the borehole wall, that is, at the interface, the acoustic wave generates a propagating electromagnetic wave (EM wave) [9]. The almost same arrival time of small wave components in full waveforms indicates that they propagate with higher speed than that of any acoustic waves.

In the spectral representation, the response allows the identification of the pesudo-Rayleigh wave (pR.1), which has an about cut-off frequency of $7 \mathrm{kHz}$. The frequency of the excited source pulse is enough to reveal this wave mode. The excitation is stronger at low frequency for Stoneley wave without cut-off frequency. Spectral representation of electric field (Figure 1(c)) $E_{z}$ shows that the borehole seismoelectric conversion dominates at low frequency. Figures 1(c) and 1(d) shows that $\mathrm{P}$ wave response may be identified easily in both spectral representation and waveforms of electric field. In this case the seismoelectric conversion efficiency of $\mathrm{P}$ wave is biggest, Stoneley wave is second.

Figure 2 shows spectral response and synthetic waveforms of acoustic waves and magnetic field $H_{r}$ for fast formation generated by an acoustic dipole source. In the spectral representations of borehole pressure (Figure 2(a)), the two flexual modes obviously appear in the frequency range $0-20 \mathrm{kHz}$; however, in the spectral representations of magnetic field, the lowest mode is dominating. From Figures 2(b) and 2(d), the waveforms of borehole pressure with 


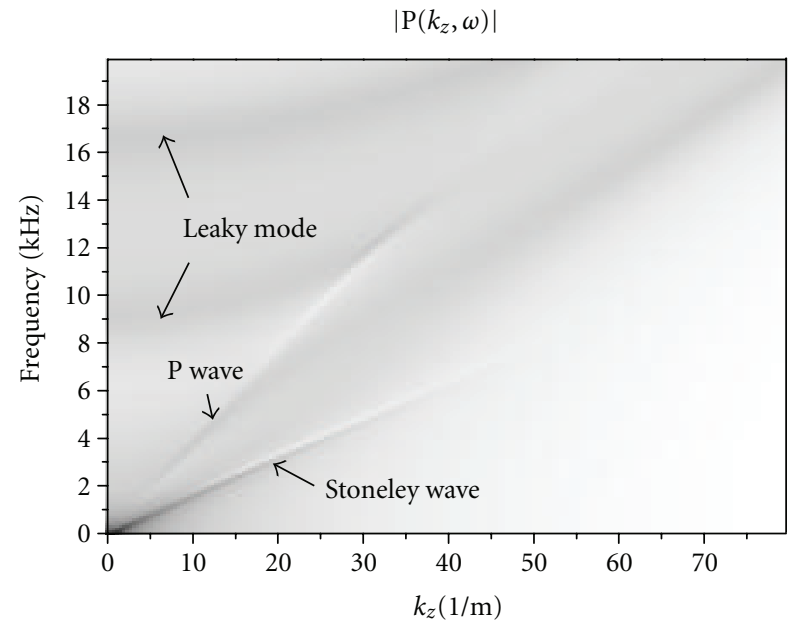

(a)

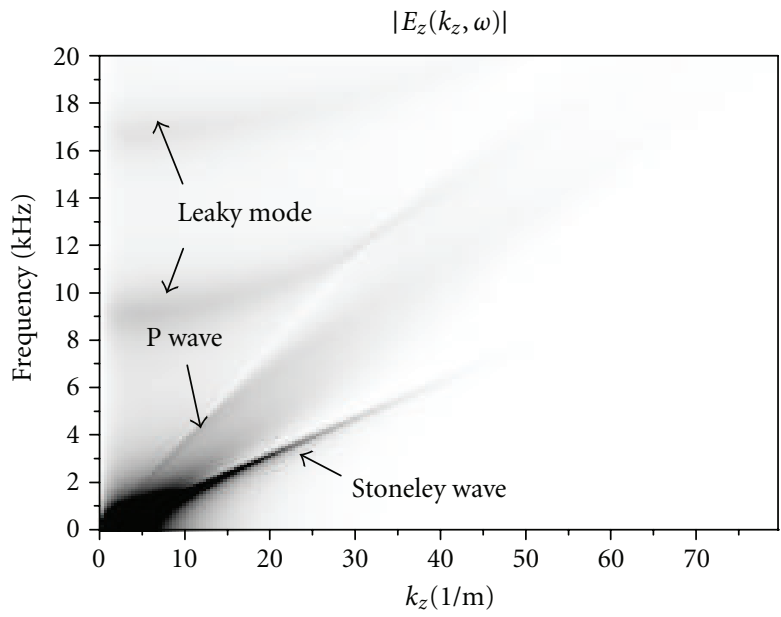

(c)

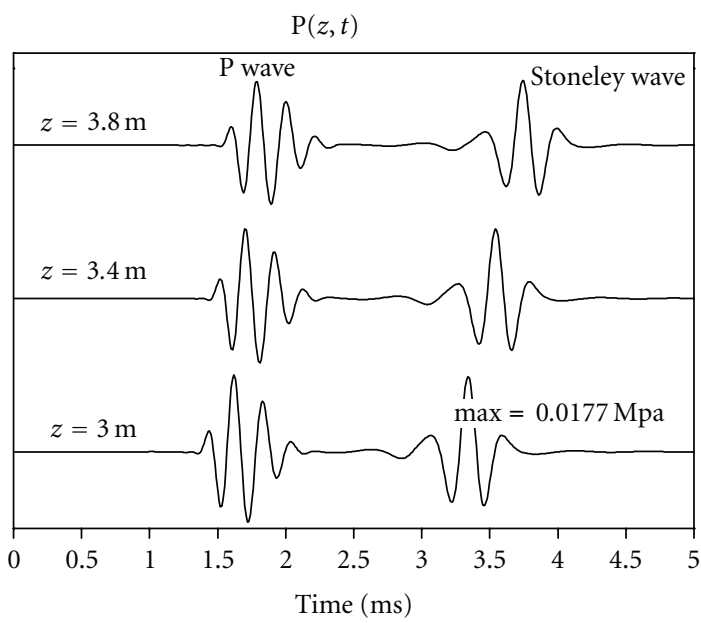

(b)

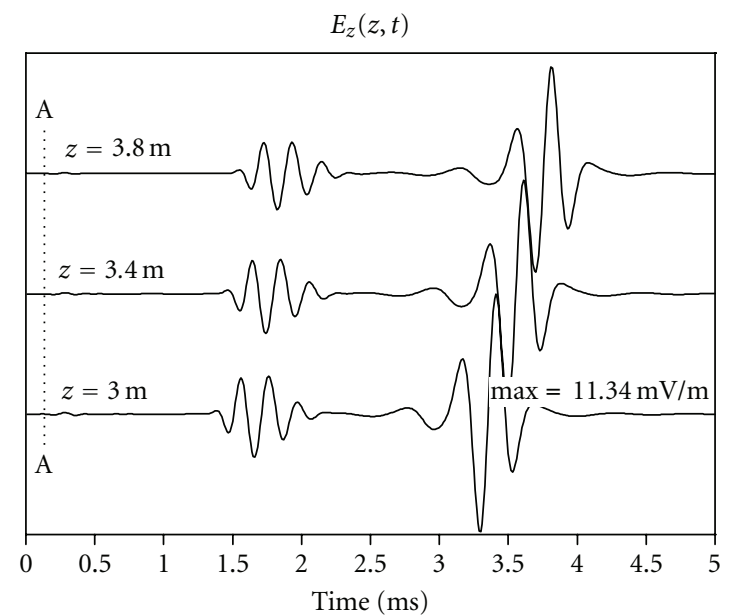

(d)

Figure 4: Spectral representation and time response of borehole pressure and electric fields for slow formation generated by monopole source. (a) and (c) are spectral of pressure and electric fields, respectively. (b) and (d) are time responses of pressure and electric fields, respectively.

$3 \mathrm{kHz}$ center frequency dominates by flexural guided waves; however, that of magnetic field has two groups with different velocity. Again, from Figure 2(d) we see a relatively weak but visible A-A group; it arrives simultaneously on all receivers. And the following groups having apparent velocities of acoustic flexural waves velocities of borehole acoustic waves.

Figure 3 shows spectral response and synthetic waveforms of acoustic waves and magnetic field $H_{r}$ for fast formation generated by an acoustic quadrupole source. In the spectral representations of borehole pressure (Figure 3(a)), the two screw modes obviously appear in the range of 0 $20 \mathrm{kHz}$; however, in the spectral representations of magnetic field, the lowest mode is dominating. From Figures 3(b) and $3(\mathrm{~d})$, the waveforms of acoustic waves quadrupole sources with the center frequency $5 \mathrm{kHz}$ mainly dominated by screw waves and that of magnetic field consists of groups having apparent velocities of acoustic screw waves. And we can find almost no EM waves in magnetic field full waveforms.
3.2. Slow Formation. Figure 4 shows spectral response and synthetic waveforms for acoustic waves and electric field for slow formation generated by an acoustic monopole source. From Figures 4(b) and 4(d), we can find there are three wave groups in electric field $E_{z}$ different from two in acoustic waveforms. A clear seismoelectric response A-A group for the electric field $E_{z}$ is relatively weak but visible in full waveforms, which were recorded at all receivers simultaneously. And the following groups for the electric field having apparent velocities are comparable to those of borehole acoustic waves.

In the spectral representation, the pesudo-Rayleigh wave disappears and the response allows the identification of $\mathrm{P}$ wave and Stoneley wave. The excitation is stronger at low frequency for Stoneley wave for slow formation same as fast formation. Spectral representation of electric field (Figure 4(c)) $E_{z}$ shows that the borehole seismoelectric conversion dominates at low frequency. Figures $4(\mathrm{c})$ and 


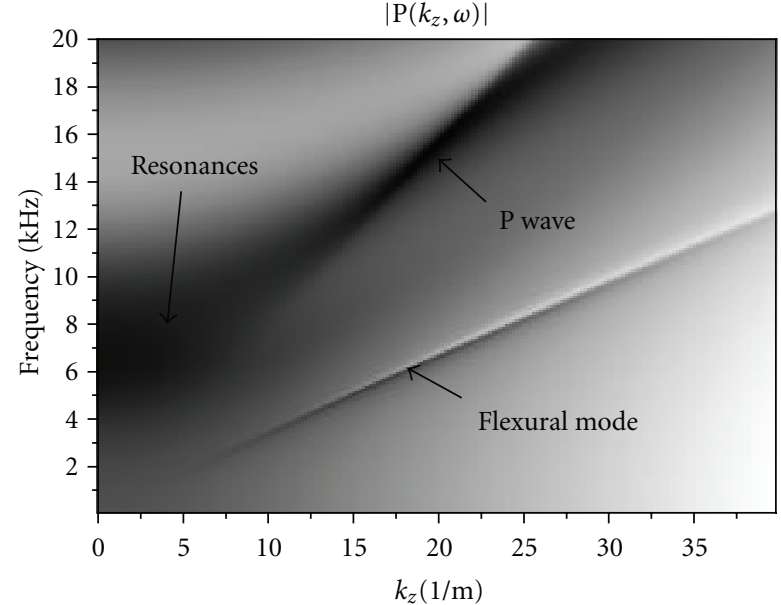

(a)

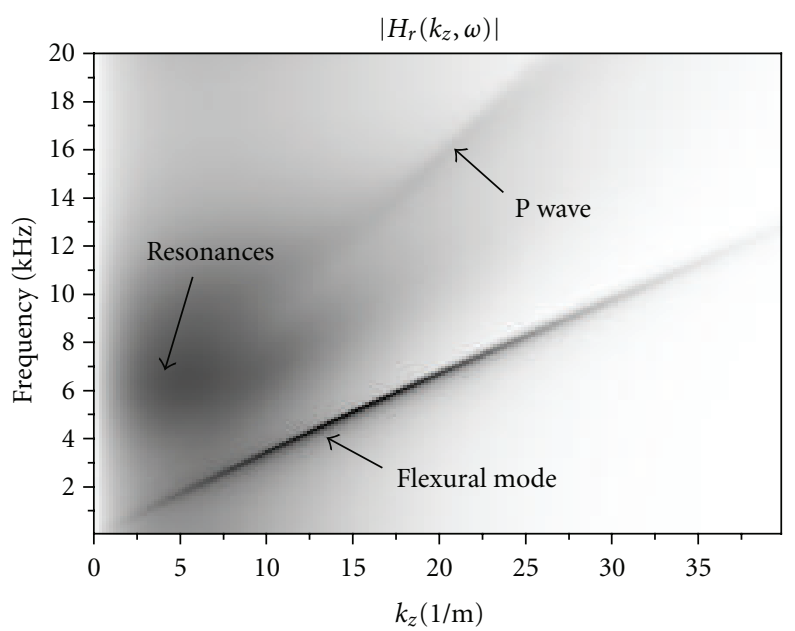

(c)

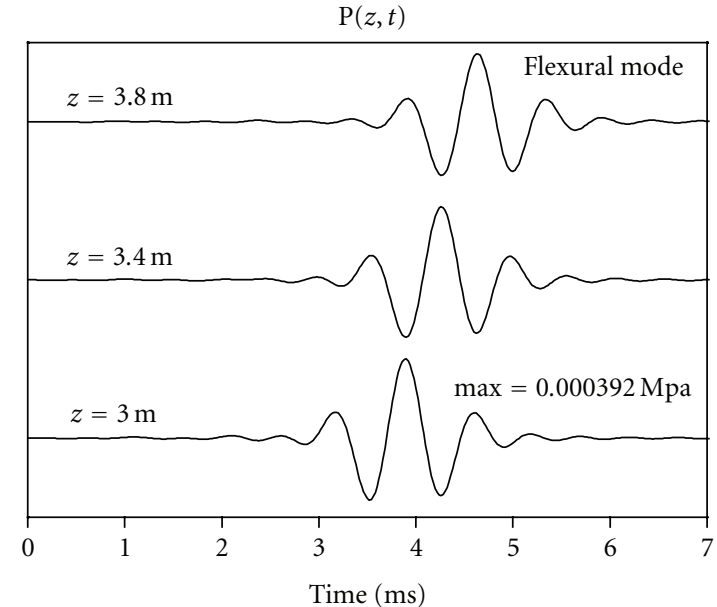

(b)

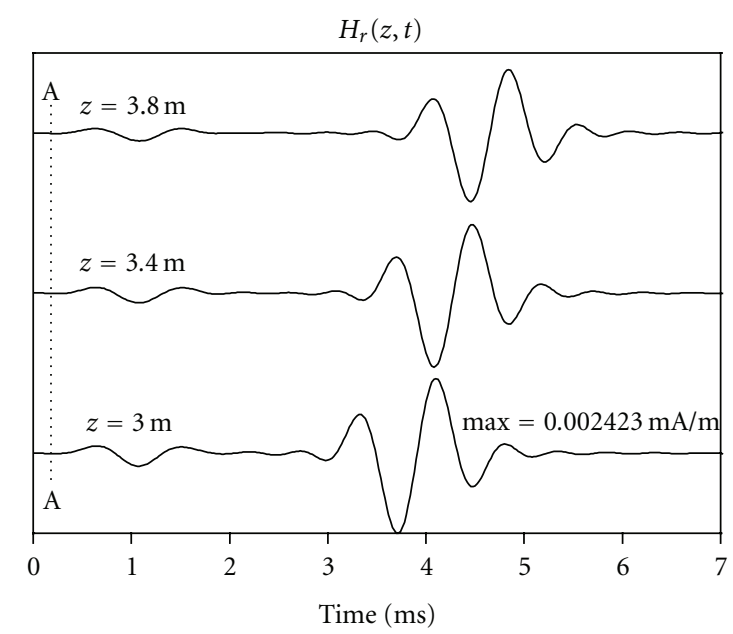

(d)

Figure 5: Spectral representation and time response of borehole pressure and magnetic fields for slow formation generated by dipole source. (a) and (c) are spectral of pressure and magnetic fields, respectively. (b) and (d) are time responses of pressure and magnetic fields, respectively.

4(d) shows that $\mathrm{P}$ wave response may be identified easily in both spectral representation and waveforms of electric field. Different from fast formation case, the seismoelectric conversion efficiency of Stoneley wave is biggest, $\mathrm{P}$ wave is second.

Figure 5 shows spectral response and synthetic waveforms of acoustic waves and magnetic field $H_{r}$ for slow formation generated by an acoustic dipole source. In the spectral representations of acoustic (Figure 5 (a)), one flexural mode appears in the frequency range $0-20 \mathrm{kHz}$, and in the spectral representations of magnetic field (Figure 5 (b)), the flexural mode is dominating. From Figures 5(b) and 5 (d), the waveforms of borehole pressure with $1 \mathrm{kHz}$ center frequency dominates by flexural guided waves; however, that of magnetic field has two groups with different velocity. Again, from Figure 5(d) we see a relatively weak but visible A-A group; it arrives simultaneously on all receivers. And the following group has apparent velocities of acoustic flexural waves velocities of borehole acoustic waves. Again, it shows the borehole seismoelectric conversion efficiency is strong at low frequency range.

Figure 6 shows spectral response and synthetic waveforms of acoustic waves and magnetic field $H_{r}$ for slow formation generated by an acoustic quadrupole source. In the spectral representations of acoustic (Figure 6(a)), a screw mode appears in the range of $0-10 \mathrm{kHz}$; however, in the spectral representations of magnetic field, this mode is more evident. From Figures 6(b) and 6(d), the waveforms of acoustic waves excited by quadrupole source with the center frequency $3 \mathrm{kHz}$ mainly dominated by screw waves and that of magnetic field consists of groups having apparent velocities of acoustic screw waves. We almost cannot find the EM wave in the full waveforms of magnetic field $H_{r}$.

\section{Conclusions and Discussions}

In this paper, multipole seismoelectric logging waveforms are simulated in a fluid-filled borehole embedded in permeable 


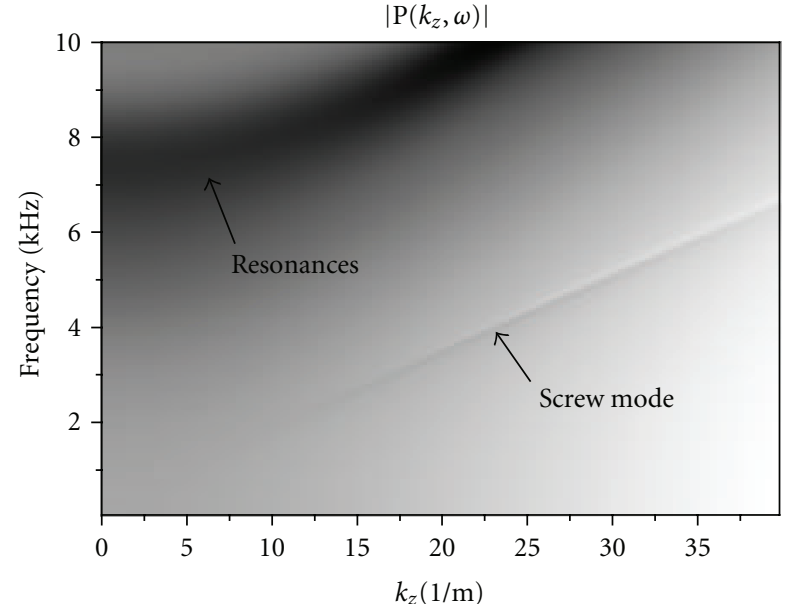

(a)

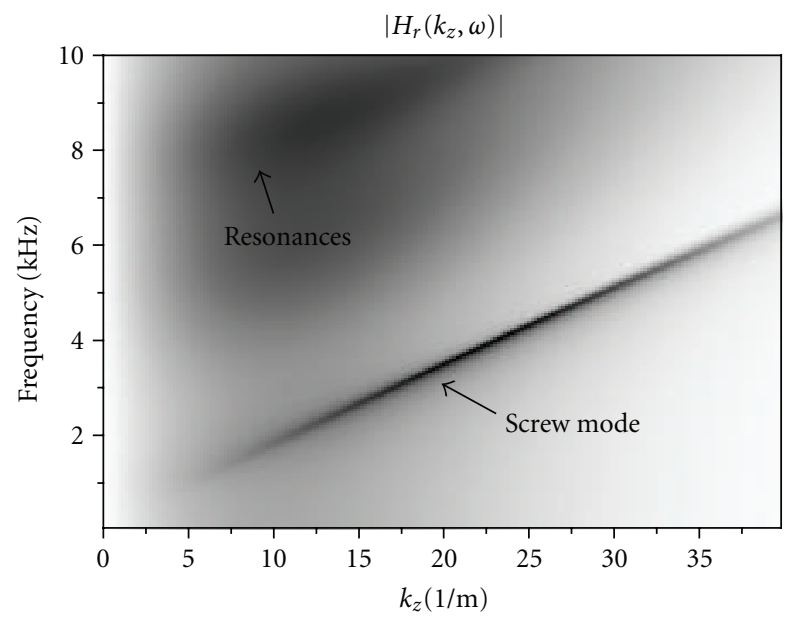

(c)

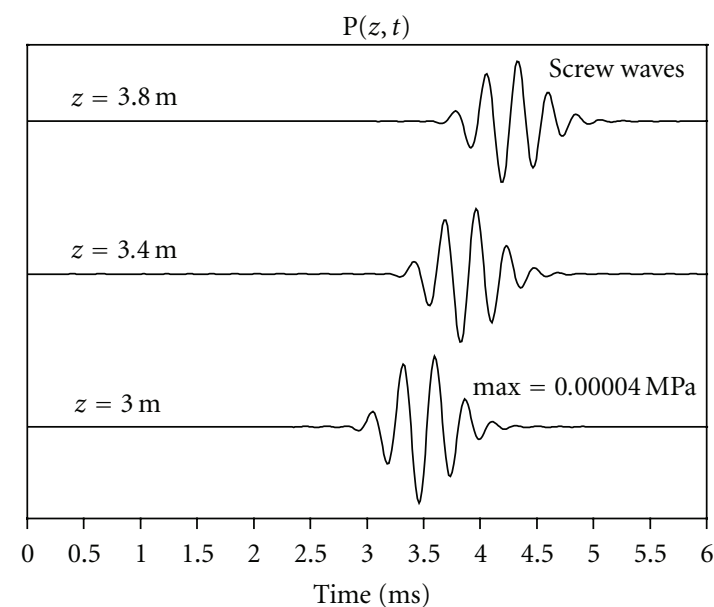

(b)

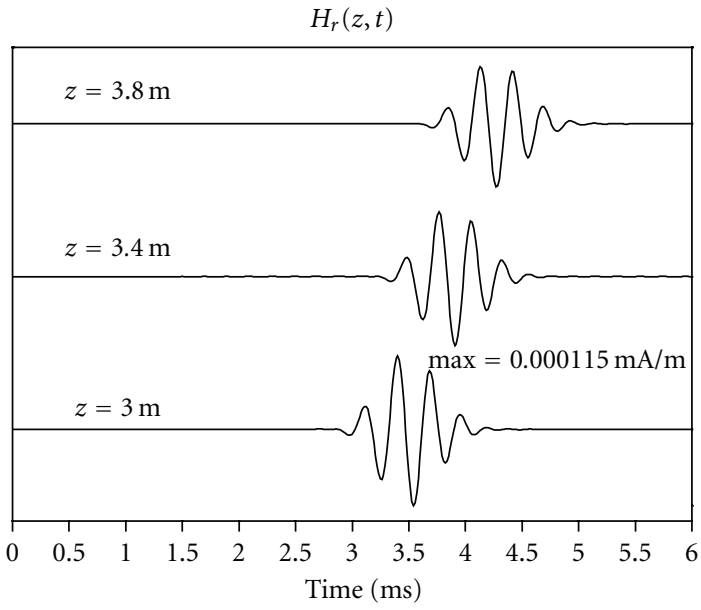

(d)

FIGURE 6: Spectral representation and time response of borehole pressure and magnetic fields for slow formation generated by quadrupole source. (a) and (c) are spectral of pressure and magnetic fields, respectively. (b) and (d) are time responses of pressure and magnetic fields, respectively.

formations based on the entire Pride seismoelectric theory. We consider the nonaxisymmetric seismoelectric field excited by acoustic multipole sources. Expressions for the multipole seismoelectric field quantities of interest were derived from the potential formulations. The full waveforms of acoustic waves and electric and magnetic fields in the time domain propagation in borehole are simulated by using a discrete wave number integration. The results show that electric and magnetic field detectors in a borehole can detect both the EM waves and the seismoelectric field. One can measure three components of electric field and magnetic field when higher order multipole sources $(n \geq 1)$ are excited. The results shows that one can measure tens of millivolt electric fields as long as one selects an acoustic source with pressure amplitude being $1 \mathrm{MPa}$ measuring $0.1 \mathrm{~m}$ from the source center. However, it is the weakness of electrical and magnetic signals in the presence of the big background noise that is one main obstacle to application of seismoelectric tools. Therefore, enhancing the signal-tonoise ratio is the key for seismoelectric logging measurement.
The amplitudes of electromagnetic field should be related to formation parameters through coupling coefficients $L(\omega)$; the seismoelectric conversion efficiencies of the Stoneley wave, the flexural guided wave and the screw guided waves are slightly different due to borehole effect in nature. The borehole seismoelectric conversion mainly depends on the relative motion between a solid and a fluid, which is generated by different acoustic modes. The relationship between borehole seismoelectric conversion efficiencies and the formation parameters needs further research in detail.

\section{Acknowledgments}

This work was supported by the National Natural Science Foundation of China (Grants nos. 40974067 and 41004044), the State Key Laboratory of Acoustics (IACAS) (Grant no. 200807) and Scientific Forefront and Interdisciplinary innovation project of Jilin University (Grant no. 200903319). 


\section{References}

[1] M. A. Biot, "Theory of propagation of elastic waves in a fluidsaturated porous solid. I-Low-frequency range," Journal of the Acoustical Society of America, vol. 28, pp. 168-178, 1956.

[2] M. A. Biot, "Theory of propagation of elastic waves in a fluidsaturated porous solid. II-High-frequency range," Journal of the Acoustical Society of America, vol. 28, pp. 179-191, 1956.

[3] T. J. Plona, "Observation of a second bulk compressional wave in a porous medium at ultrasonic frequencies," Applied Physics Letters, vol. 36, no. 4, pp. 259-261, 1980.

[4] M. A. Biot, "Generalized theory of acoustic propagation in porous dissipative media," Journal of the Acoustical Society of America, vol. 34, pp. 1254-1264, 1962.

[5] S. R. Pride and S. Garambois, "The role of Biot slow waves in electroseismic wave phenomena," Journal of the Acoustical Society of America, vol. 111, no. 2, pp. 697-706, 2002.

[6] H. S. Hu, "Acoustic head wave on the borehole wall in a porous formation and the causes for its accompanying electromagnetic field," Acta Physica Sinica, vol. 52, pp. 19511959, 2003.

[7] A. Thompson and G. Gist, "Geophysical applications of electrokinetic conversion," The Leading Edge, vol. 12, pp. 1169-1173, 1993.

[8] S. Pride, "Governing equations for the coupled electromagnetics and acoustics of porous media," Physical Review B, vol. 50, no. 21, pp. 15678-15696, 1994.

[9] S. R. Pride and M. W. Haartsen, "Electroseismic wave properties," Journal of the Acoustical Society of America, vol. 100, no. 3, pp. 1301-1315, 1996.

[10] M. W. Haartsen and S. R. Pride, "Electroseismic waves from point sources in layered media," Journal of Geophysical Research B, vol. 102, no. 11, pp. 24745-24769, 1997.

[11] O. V. Mikhailov, J. Queen, and M. N. Toksöz, "Using borehole electroseismic measurements to detect and characterize fractured (permeable) zones," Geophysics, vol. 65, no. 4, pp. 1098 $1112,2000$.

[12] Z. Zhu, M. W. Haartsen, and M. N. Toksöz, "Experimental studies of electrokinetic conversions in fluid-saturated borehole models," Geophysics, vol. 64, no. 5, pp. 1349-1356, 1999.

[13] Z. Zhu and M. N. Toksöz, "Crosshole seismoelectric measurements in borehole models with fractures," Geophysics, vol. 68, no. 5, pp. 1519-1524, 2003.

[14] Z. Zhu and M. N. Toksöz, "Seismoelectric and seismomagnetic measurements in fractured borehole models," Geophysics, vol. 70, no. 4, pp. F45-F51, 2005.

[15] H. S. Hu, K. X. Wang, and J. N. Wang, "Simulation of acoustically induced electromagnetic field in a borehole embedded in a porous formation," Tech. Rep. 13, Earth Resources Laboratory of MIT, 2000.

[16] A. L. Kurkjian and S. K. Chang, "Acoustic multipole sources in fluid boreholes," Geophysics, vol. 51, no. 1, pp. 148-163, 1986.

[17] Z. W. Cui, Theoretical and numerical study of modified Biot's models, acoustoelectric well logging and acoustic logging while drilling excited by multipole acoustic sources, Ph.D. thesis, Jilin University, Jilin, China, 2004.

[18] D. P. Schmitt, Y. Zhu, and C. H. Cheng, "Shear wave logging in semi-infinite saturated porous formation," Journal of the Acoustical Society of America, vol. 84, pp. 2230-2244, 1988.

[19] B. Zhang, K. Wang, and Q. Dong, "Nonaxisymmetric acoustic field excited by a cylindrical tool placed off a borehole axis and extraction of shear wave," Journal of the Acoustical Society of America, vol. 99, no. 2, pp. 682-690, 1996.
[20] Z. W. Cui, K. X. Wang, H. S. Hu, and J. G. Sun, "Acoustoelectric well logging by eccentric source and extraction of shear wave," Chinese Physics, vol. 16, no. 3, pp. 746-752, 2007.

[21] W. L. Roever, J. H. Rosenbaum, and T. F. Vining, "Acoustic waves from an impulsive source in a fluid-filled borehole," Journal of the Acoustical Society of America, vol. 55, no. 6, pp. 1144-1157, 1974.

[22] W. Guan, H. S. Hu, and Z. T. Chu, "Formulation of the acoustically-induced electromagnetic field in a porous formation in terms of Hertz vectors and simulation of the borehole electromagnetic field excited by an acoustic multipole source," Acta Physica Sinica, vol. 55, no. 1, pp. 267-274, 2006.

[23] D. P. Schmitt and M. Bouchon, "Full-wave acoustic logging: synthetic microseismograms and frequency-wavenumber analysis," Geophysics, vol. 50, no. 11, pp. 1756-1778, 1985. 

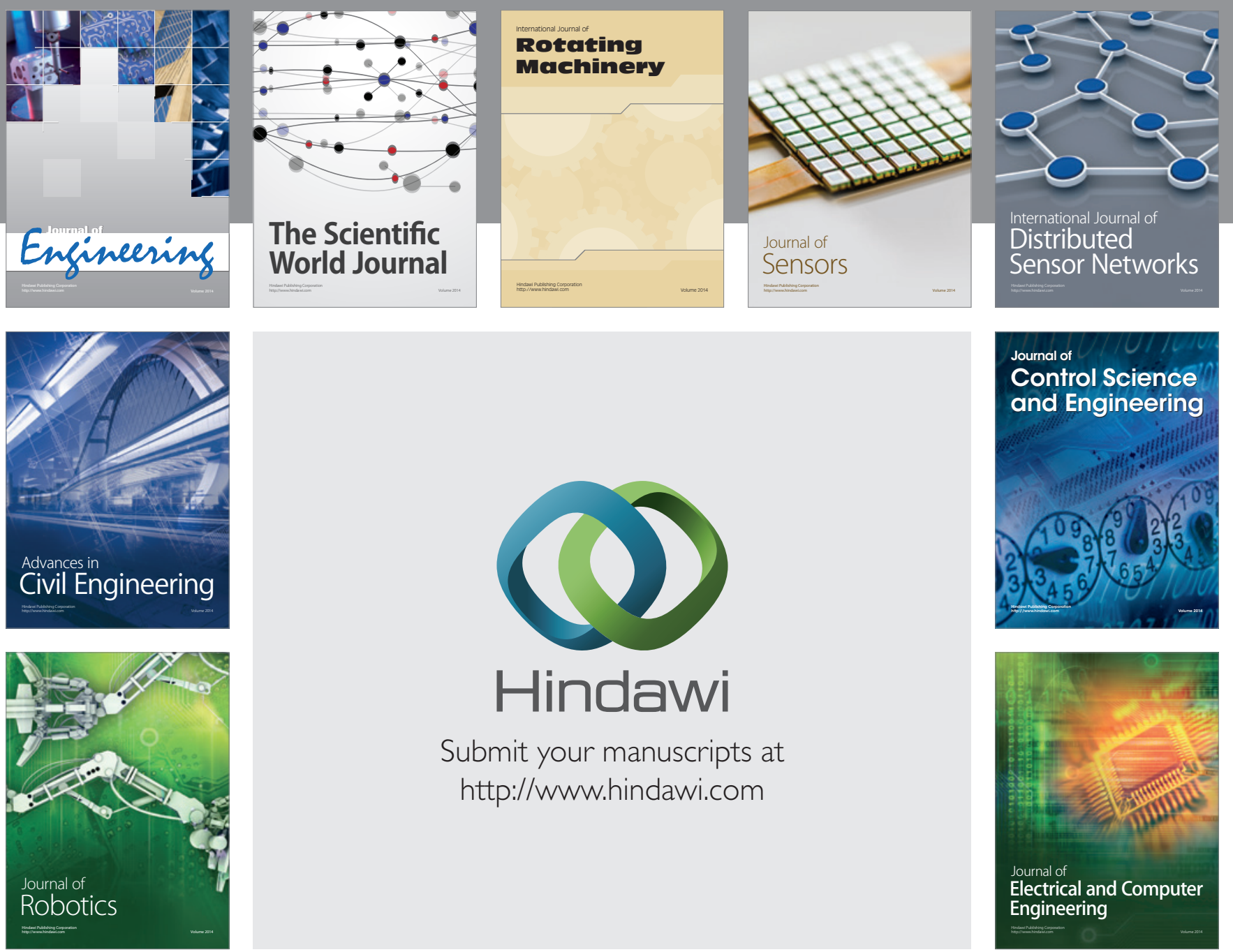

Submit your manuscripts at

http://www.hindawi.com
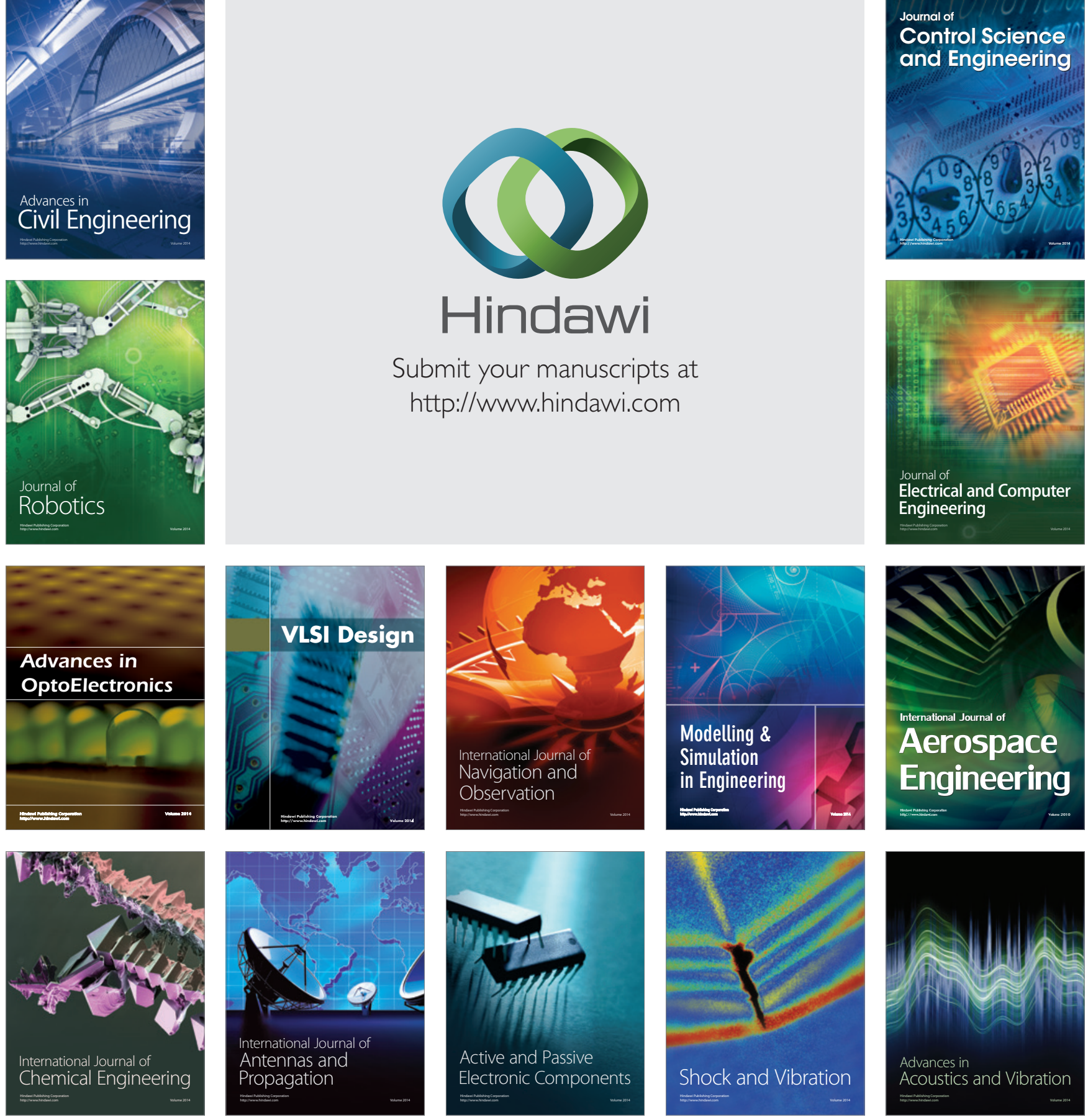